Check for updates

Cite this: Phys. Chem. Chem. Phys.

2021, 23, 4262

\title{
Shape changes and budding of giant vesicles induced by an internal chemical trigger: an interplay between osmosis and $\mathrm{pH}$ change $\dagger$
}

\author{
Gábor Holló, $\ddagger^{a}$ Ylenia Miele $\ddagger^{\mathrm{b}}$ Federico Rossi (iD *c and István Lagzi (D) *d
}

\begin{abstract}
Shape transformation and budding of phospholipid/fatty acid giant hybrid vesicles can be induced by an internal chemical stimulus ( $\mathrm{pH}$ change) when coupled with an osmotic shock. In particular, an autocatalytic enzymatic reaction set (urea-urease system), confined in the lumen of 1-palmitoyl-2-oleoyl-sn-glycero-3phosphocholine (POPC)/oleic acid ( $\mathrm{HOA}$ ) vesicles, can force the budding of the hosting vesicle, when properly fed by a trans-membrane substrate infusion. Herein, we elucidate the budding mechanism by simulating the shape changes of a vesicle during the enzymatic reaction. The area-difference-elasticity (ADE) theory is thus implemented to minimize the surface elastic energy and obtain the equilibrium shape at different values of the reduced volume and different values of the reduced preferred area difference $\left(\Delta a_{0}\right)$. Simulations, together with control experiments, unambiguously show that to obtain an effective vesicle shape transformation, the osmotic stress and the $\mathrm{pH}$ change in the lumen of the vesicle must act in synergy at the same timescale. Osmotic pressure induces a vesicle deflation (volume loss), while the $\mathrm{pH}$ change affects the preferred area difference between the outer and the inner membrane leaflets.
\end{abstract}

Received 16th November 2020 Accepted 1st February 2021

DOI: $10.1039 / \mathrm{d} 0 \mathrm{cp} 05952 \mathrm{~h}$

rsc.li/pccp we know, is a complex mechanism resulting from a long story of natural evolution, ${ }^{15}$ it is thus plausible that such a complex mechanism might be evolved, in prebiotic age, from separate processes such as membrane shape transformation, autonomous self-copying of complex molecules and self-division processes, the latter intended as the splitting of a mother cell without the addition of membrane forming molecules. The trigger of the shape transformation of vesicles, incorporating their self-division, can be categorized into two types of approaches, externally and internally triggered shape transformations. In most studies in synthetic and artificial systems, the deformation and division processes of vesicles were obtained by external triggers (e.g., the addition of the membrane precursors to the solution, changing the temperature, etc.), ${ }^{16-23}$ even though in biological systems the division is an autonomous process driven by internal mechanism of the cells. ${ }^{24}$ Therefore, engineering and design of artificial systems having internally trigger mechanisms for the shape transformation are more relevant, elegant and challenging approaches that require complex thinking involving non-trivial design steps. ${ }^{25}$ In the approach of Sugawara and his coworkers, the division process of giant unilamellar vesicles (GUVs) was mediated by the interplay of the polymerase chain reaction taking place in the lumen of vesicles and the subsequent addition of the membrane precursors to the system. ${ }^{26}$ The division rate of the vesicles was significantly lower in the absence of the enzymatic reaction. In another interesting study, a protein system was encapsulated into 
the lumen of GUVs and used to periodically control the shape transformation of GUVs from pear shape to dumbbell shape and to drive budding and merging of the buds with the mother vesicles. ${ }^{27}$ A novel approach demonstrated that deformation of GUVs shape can also be induced by purely physical stimuli such as inner hydrodynamic forces and mechanical torques generated by active nanoparticles. ${ }^{28,29}$

Recently, ${ }^{30}$ we have reported an internally triggered mechanism, in which mixed 1-palmitoyl-2-oleoyl-sn-glycero-3phosphocholine (POPC)/oleic acid (HOA) pH-responsive GUVs (with size ranging between 5 and $50 \mu \mathrm{m}$ ) underwent shape deformations triggered by an autocatalytic enzymatic (ureaurease) reaction ${ }^{31-35}$ encapsulated inside the vesicles and coupled to a cross-membrane transport of the substrate, urea. The deformations included the following shapes: prolate, pear shape and buds (two vesicles connected by a narrow neck), and we speculated that the deformation dynamics was due to the interplay between an osmotic shock and the $\mathrm{pH}$ change taking place in the lumen of the GUVs.

In this work, we thoroughly investigate the role of the $\mathrm{pH}$ change and that of the osmotic shock to clarify and model the budding mechanism. Moreover, we interpret the shape changes observed during the urea-urease reaction with the areadifference elasticity (ADE) theory that can be applied to membranes of fixed topology, and therefore it allows to predict the formation of buds but not the membrane rupture and the complete self-division. According to this theory, the morphology of a vesicle with surface area $A$ and volume $V$ is determined by two geometrical parameters, the reduced volume $\nu$ and the normalized preferred area difference of the two monolayers $\Delta a_{0}{ }^{36}$ The ADE model has been successfully applied to investigate, among the others, shape changes in binary vesicles of DLPE (1,2-dilauroyl-sn-glycero-3-phosphoethanolamine) and DPPC (1,2-dipalmitoyl-sn-glycero-3-phosphocholine) during heating-cooling cycles ${ }^{21}$ and shape changes in pure fatty acid vesicles fed externally with $\mathrm{NaOH} .{ }^{37}$ Herein, we propose a model that integrate the $\mathrm{pH}$ changes due to the enzymatic reaction with the chemistry of fatty acids and takes into account the osmotic shock to explain the $\nu$ and $\Delta a_{0}$ changes which cause the vesicles shape transformations.

\section{Experimental methods}

\subsection{Preparation and analysis of giant lipid vesicles}

For GUVs preparation and encapsulation of chemical species, the phase transfer method ${ }^{34,38-40}$ was used, which allowed full encapsulation of chemical species (details are reported in ESI $\dagger$ ). Briefly, the method consists in the stratification of a water-inoil emulsion (enzyme urease, acetic acid and sucrose are dissolved in the water phase) over an aqueous phase (outer solution containing glucose and acetic acid) separated by an interface of amphiphilic molecules mixed in different proportions (1-palmitoyl-2-oleoyl-sn-glycero-3-phosphocholine, POPC and oleic acid, HOA). The formation of the vesicles takes place at the interface of the two phases, where emulsion droplets sink by gravity in the outer phase and get coated by the amphiphilic molecules to form bilayers.

To observe the shape transformation dynamics, vesicles were added with $10 \mu \mathrm{L}$ of a solution containing [urea] $=180 \mathrm{mM}$, [glucose $]=200 \mathrm{mM}$ glucose and $\left[\mathrm{CH}_{3} \mathrm{COOH}\right]=1 \times 10^{-3} \mathrm{mM}$ to trigger the division. Samples were investigated by an epifluorescence microscope (ORMATEK TL-INV 100) by taking images every $0.5 \mathrm{~s}$ with a CMOS camera (PIXELINK PL-D755CU) both in visible and in fluorescence $\left(\lambda_{\mathrm{ex}}=450 \mathrm{~nm}\right.$ and $\left.\lambda_{\mathrm{em}}=510 \mathrm{~nm}\right)$ mode. Fluorescence intensity was used to characterise the $\mathrm{pH}$ change inside the vesicles. Recorded images were analysed by means of ImageJ software. ${ }^{41}$

\subsection{Simulations of the deformation of vesicles}

The software Surface Evolver $2.70^{42}$ was used to compute the relaxed vesicle shape by minimizing the energy functional $W$, i.e. the Helfrich energy, ${ }^{43,44}$ complemented with a term that, according to the ADE theory, accounts for the area difference between the outer and inner leaflets of the lipid membrane, ${ }^{45,46}$ expressed by

$$
W=\frac{1}{2} \kappa_{\mathrm{c}} \oint_{S}\left(C_{1}+C_{2}-C_{0}\right)^{2} \mathrm{~d} A+\frac{\kappa_{\mathrm{r}}}{2 A_{0} h^{2}}\left(\Delta A-\Delta A_{0}\right)^{2}
$$

where $C_{1}$ and $C_{2}$ are the local principal curvatures, $C_{0}$ is the local spontaneous curvature and the integral is calculated on the whole surface $(S)$ of the vesicles, ${ }^{47,48} \kappa_{\mathrm{c}}$ and $\kappa_{\mathrm{r}}$ are the local and the non-local bending moduli, respectively, $A$ is the vesicle area. The spontaneous curvature depends on the shape of the membrane constituents. POPC molecules are approximately cylinder-shaped, which makes their intrinsic curvature close to zero and the bilayer structures are flat. ${ }^{49}$ Protonated oleic acid molecules have a slightly negative intrinsic curvature, ${ }^{50}$ while deprotonated oleic acid molecules have a positive curvature. In mixed phospholipid/oleic acid/oleate bilayers, the average spontaneous curvature of a membrane composed of phospholipids and roughly equal amounts of oleic acid and oleate $\left(\mathrm{pH} \sim \mathrm{p} K_{\mathrm{a}}\right)$ is expected to be close to zero $\left(C_{0}=0\right) .{ }^{18}$ The area difference between the inner and the outer leaflets $(\Delta A)$ is generally calculated from the integral of the mean curvature

$$
\Delta A=h \oint_{S}\left(C_{1}+C_{2}\right) \mathrm{d} A
$$

where $h$ is the distance between neutral surfaces of both bilayer leaflets, however, in our case, $\Delta A$ is calculated directly from the areas extracted from experimental images.

$\Delta A_{0}$ is the preferred area difference and is given by

$$
\Delta A_{0}=\left(N_{\text {outer }}-N_{\text {inner }}\right) \tilde{a}
$$

where $N_{\text {outer }}$ and $N_{\text {inner }}$ are the number of molecules in the outer and inner leaflet, respectively, $\tilde{a}$ is the cross-sectional area of the amphiphiles. Thus, $\Delta A_{0}$ depends on the composition of the two membrane monolayers and their interaction with the surroundings, $\Delta A$ depends on the shape. ${ }^{45}$ Dividing for the bending energy of a sphere $\left(8 \pi \kappa_{\mathrm{c}}\right)$, the reduced energy is 
obtained

$$
w=\frac{1}{4} \oint_{S}\left(c_{1}+c_{2}\right)^{2} \mathrm{~d} a+\frac{\kappa_{\mathrm{r}}}{\kappa_{\mathrm{c}}}\left(\Delta a-\Delta a_{0}\right)^{2}
$$

where $\mathrm{d} a=\mathrm{d} A / 4 \pi R_{\mathrm{s}}, c_{1}=C_{1} R_{\mathrm{s}}, c_{2}=C_{2} R_{\mathrm{s}}$ and the reduced area differences are $\Delta a=\Delta A / 8 \pi h R_{\mathrm{S}}$ and $\Delta a_{0}=\Delta A_{0} / 8 \pi h R_{\mathrm{s}}$ and $R_{\mathrm{s}}$ is the radius of the sphere. The ratio $\kappa_{\mathrm{r}} / \kappa_{\mathrm{c}}$ can be infinitely large or can tend to zero. When the ratio is very large the limit is called the "strict bilayer couple" model (BC) and was originally introduced by the requirement of incompressible membrane monolayers leading to the relationship $\Delta a=\Delta a_{0}$. The limiting case in which the ratio $\kappa_{\mathrm{r}} / \kappa_{\mathrm{c}}$ has the value of zero is called "spontaneous curvature model" (SC). The ADE theory and the limiting cases are discussed in more detail in the following ref. 45 and 51-53. In this work, the eqn (4) was minimized numerically and the ratio $\kappa_{\mathrm{r}} /$ $\kappa_{\mathrm{c}}=3$ was chosen since this value is commonly used to describe phospholipid vesicle morphologies. $^{21,45}$

To describe the equilibrium shape of a vesicle at the minimum of the elastic energy, only two parameters can be employed, namely the reduced preferred area difference $\left(\Delta a_{0}\right.$, as previously defined) and the reduced volume $(\nu)$. If an equivalent sphere is defined by $R=\sqrt{A / 4 \pi}$, where $A$ is the surface of the vesicle, then the reduced volume can be written as

$$
\nu=\frac{V}{4 / 3 \pi R^{3}}
$$

During the energy minimization, the parameters $\Delta a_{0}$ and $v$ were constrained to set a given reduced volume $v$ and reduced area $\Delta a$, extracted from experimental images.

Radii and geometrical parameters of the vesicles were extracted from experimental frames with the help of ImageJ. The values of the volume $V$ and the surface area $A$, were calculated by means of the equations reported in the ESI $\dagger$ for each of the shape assumed by the vesicles (sphere, prolate, pear shape and budded limiting shape).

\section{Results and discussion}

\subsection{Conditions for successful divisions}

In a typical experiment, a fast enzymatic reaction inducing a $\mathrm{pH}$ change inside the GUVs with the consequent division of the vesicles, was obtained in the following conditions: urease $(0.5-$ $\left.1.5 \mathrm{U} \mathrm{mL}^{-1}\right)$, pyranine $\left(5.0 \times 10^{-5} \mathrm{M}\right)$ and acetic acid $\left(1 \times 10^{-6}\right.$ $\mathrm{M})$ were encapsulated in hybrid giant POPC/HOA vesicles with $[\mathrm{POPC}]=2.6 \mathrm{mM}$ and $[\mathrm{HOA}]=2.4 \mathrm{mM}$, urea with a final concentration after mixing of $6.0 \times 10^{-2} \mathrm{M}$ was added to the vesicles suspension to initiate the reaction. Urea penetrated into the vesicles and initiated the urea-urease enzymatic reaction, which caused a pH increase according to the scheme

$$
\mathrm{CO}\left(\mathrm{NH}_{2}\right)_{2}+\mathrm{H}_{2} \mathrm{O} \stackrel{\text { urease }}{\longrightarrow} 2 \mathrm{NH}_{3}+\mathrm{CO}_{2}
$$

The low concentration of acetic acid and the high concentration of urea ensured a clock time (i.e. the beginning of the autocatalytic $\mathrm{pH}$ increase) within 30 seconds after the addition of the urea substrate with a pH change of the order of 0.5-0.6 units. Fig. 1a reports a typical division process: starting from a spherical shape, the GUV elongates in a prolate form, assumes a pear shape and eventually divides into two daughter vesicles. We found that the self-division process occurred with a probability of $\sim 25 \%$ irrespective of the size of GUVs. The division percentage within samples in the same experimental conditions may unpredictably vary because of the uneven distribution of solutes and amphiphiles (e.g., not all the vesicles in a specimen may contain the $\mathrm{pH}$-sensitive $\mathrm{HOA}$ in the membrane), intrinsic to the phase transfer method; for this reason the concentrations of amphiphiles along the text are always intended as those initially mixed in the mineral oil phase. ${ }^{54,55}$ By means of epifluorescent microscopy, the correlation between the division and a $\mathrm{pH}$ change of the aqueous lumen was established, in particular, it was confirmed that the stages of the division process are correlated with the increase of $\mathrm{pH}$ inside the vesicles. The recorded $\mathrm{pH}$ increase was about a half unit starting from $\mathrm{pH}=6.0$ and ending at $\mathrm{pH}=6.5$. After the budding process, the vesicles did not separate from each other and remain connected by a narrow neck, however when the budded vesicles were irradiated by a laser beam, division readily took place. This is in agreement with the studies showing that fission and fusion of the vesicles are activated processes with an energy barrier ranging from 20 to $150 k_{\mathrm{B}} T$, and in cells, these processes are activated by proteins. ${ }^{56}$ FRAP (fluorescence recovery after photobleaching) experiments revealed that the two lumens of the daughter vesicles were completely isolated, but their membranes were connected by a common neck. ${ }^{30}$

To investigate the mechanism of the self-division of the GUVs, we carried out several control experiments. Fig. 2 briefly summarizes our findings. Successful divisions happen in the presence of the enzyme and substrate in the osmotic concentration gradient when the clock (induction) time of the enzymatic reaction was a few ten seconds. Buffered media annihilated the effect of the $\mathrm{pH}$ change, and no division was observed. Similarly, in the absence of either enzyme or substrate, no division was also detected. Moreover, in the absence of both, enzyme and substrate, and in the absence of the osmotic concentration gradient (but having enzyme and substrate in the system), no division was observed. Based on these control experiments, we can draw a conclusion that the $\mathrm{pH}$ change and initial osmotic concentration gradient are important ingredients of the division process. Moreover, we observed no division when the clock time of the enzymatic reaction was adjusted to $\sim 2$ min by increasing the concentration of the acetic acid in the vesicles even if the initial osmotic concentration gradient was present. From these observations, we can conclude that neither the osmotic concentration gradient nor the $\mathrm{pH}$ change alone can induce division. They must act in synergy, approximately at the same time scale.

\subsection{Numerical simulations}

To rationalize the shape transformation dynamics triggered by the enzymatic reaction in mixed POPC/HOA vesicles, we performed two types of numerical simulations: first, we minimized the energy functional $W$ (eqn (1)) of the structures reported 

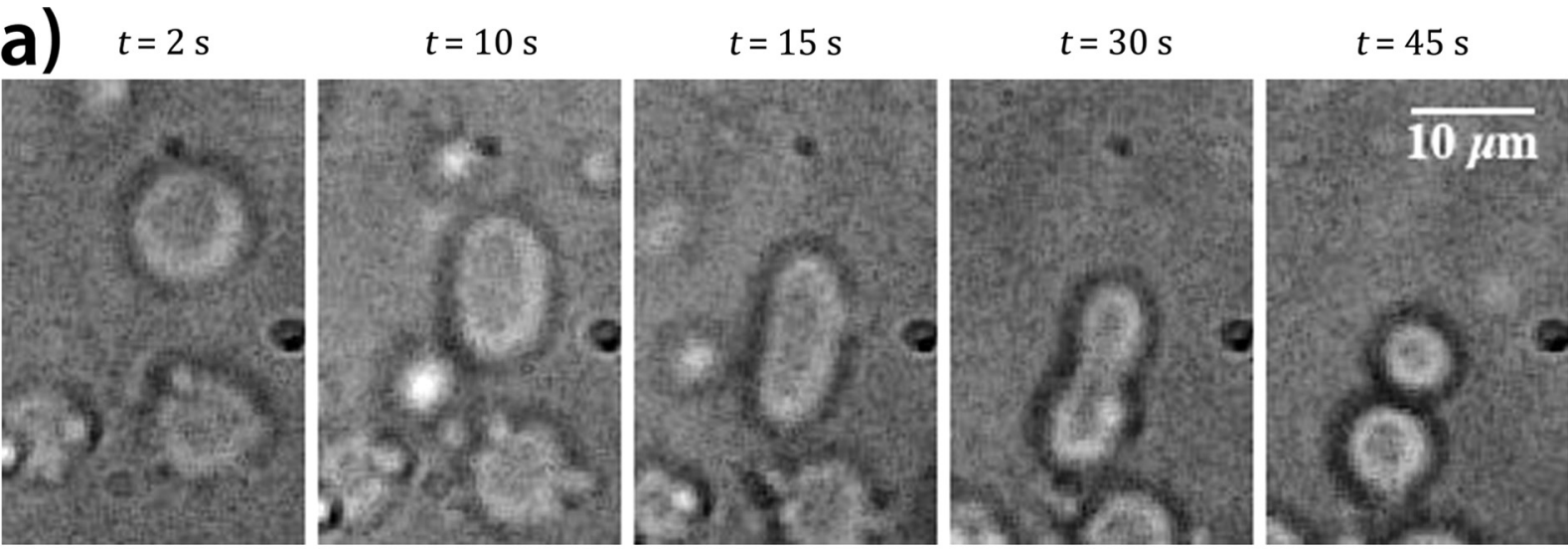

b)
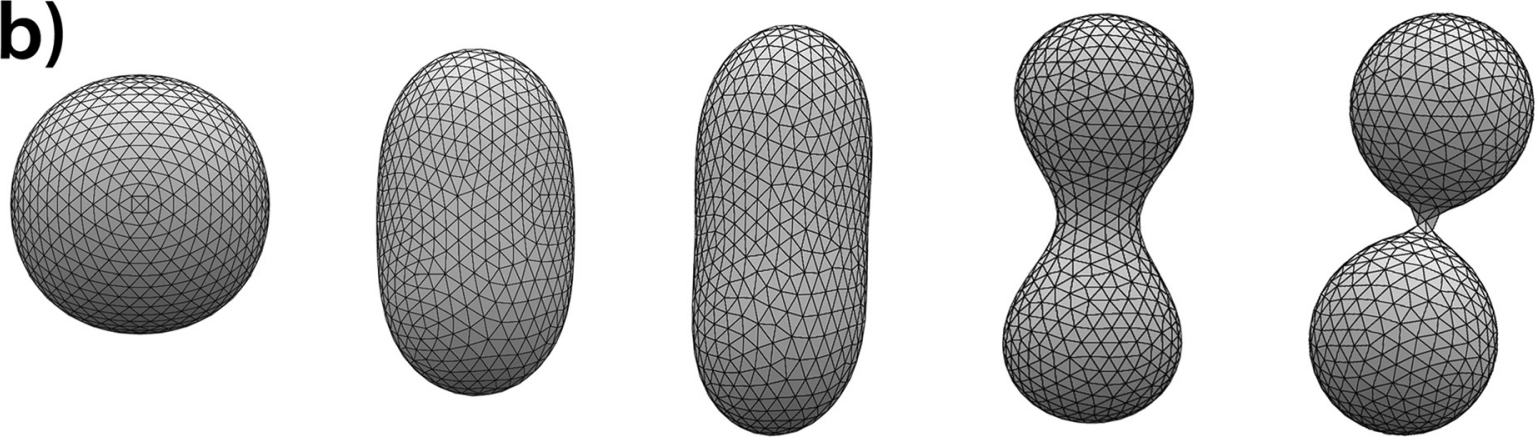

$$
\begin{aligned}
& v=1.0 \\
& \Delta \mathrm{a}_{0}=1.0
\end{aligned}
$$$$
v=0.919
$$

$\Delta \mathrm{a}_{0}=1.018$

$$
\begin{aligned}
& v=0.867 \\
& \Delta \mathrm{a}_{0}=1.037
\end{aligned}
$$

$$
\begin{aligned}
& v=0.7 \\
& \Delta \mathrm{a}_{0}=1.60
\end{aligned}
$$$$
v=0.7
$$$$
\Delta \mathrm{a}_{0}=1.68
$$

Fig. 1 Self-division of a GUV. (a) Shape transformation of a vesicle governed by the urea-urease enzymatic reaction. (b) Numerically simulated equilibrium shape structures of a vesicle using the Surface Evolver software. In the simulations, both parameters, $\nu$ (reduced volume), $\Delta a_{0}$ (reduced preferred area difference) were changed. The shape transformation starts from a spherical shape through prolate and pear shapes into two daughter vesicles.

in Fig. 1(a) at given values of $\nu$ and $\Delta a_{0}$, with the help of the software Surface Evolver; second, we implemented a kinetic model to account for the changes in the reduced preferred area difference $\left(\Delta a_{0}\right)$, following the $\mathrm{pH}$ change inside the vesicles.

Fig. 1(b) reports a typical shape deformation trajectory in the $\Delta a_{0}-\nu$ phase space, where the values of the reduced volume and the reduced preferred area difference were adjusted to match the $\Delta a-\nu$ values extracted from the experimental snapshots of Fig. 1(a). Starting from a spherical shape $\left(\Delta a_{0}=1, \nu=1\right)$, the volume decreases due to the initial osmotic shock while the area difference remains almost constant until the vesicle reaches a prolate shape at about $t=15 \mathrm{~s}\left(\Delta a_{0}=1.037, \nu=\right.$ 0.867 ); when the enzymatic reaction raises abruptly the $\mathrm{pH}$ during the autocatalytic formation of ammonia and carbon dioxide, the solubilisation of oleate molecules in the inner leaflet of the membrane causes $\Delta a_{0}$ to significantly increase driving the vesicle to the budded limiting shape $\left(t \sim 45 \mathrm{~s}, \Delta a_{0}=1.68, \nu=\right.$ 0.7). During the last phase the reduced volume decreases more slowly since the osmotic pressure is smoothed out by the permeation of the urea from the outside to the vesicle lumen.

To rationalise the shape transformation dynamics of the vesicles we propose a kinetic model based on 7 main chemical species, namely urea (S, substrate), urease (E, enzyme), pyranine (fluorescent dye, pyrOH), oleic acid (HOA), acetic acid (HA), ammonia
$\left(\mathrm{NH}_{3}\right)$ and carbon dioxide $\left(\mathrm{CO}_{2}\right)$. Some of these chemical species can be located outside of the vesicles having cross membrane properties. The $\mathrm{pH}$ inside and outside the vesicles is governed by the following equilibria

$$
\begin{aligned}
& \mathrm{CO}\left(\mathrm{NH}_{2}\right)_{2}+\mathrm{H}_{2} \mathrm{O} \stackrel{\text { urease }}{\longrightarrow} 2 \mathrm{NH}_{3}+\mathrm{CO}_{2} \\
& \mathrm{NH}_{4}^{+} \stackrel{k_{2}}{\rightleftarrows} \mathrm{NH}_{3}+\mathrm{H}^{+} \\
& \mathrm{CO}_{2}+\mathrm{H}_{2} \mathrm{O} \stackrel{k_{3}}{\rightleftarrows} \mathrm{H}^{+}+\mathrm{HCO}_{3}^{-} \\
& \mathrm{HCO}_{3} \stackrel{\stackrel{k_{4}}{\rightleftarrows}}{\rightleftarrows} \mathrm{H}^{+}+\mathrm{CO}_{3}^{2-} \\
& \mathrm{H}_{2} \mathrm{O} \stackrel{k_{5}}{\rightleftarrows} \mathrm{H}^{+}+\mathrm{OH}^{-} \\
& \mathrm{HA} \stackrel{k_{6}}{\rightleftarrows} \mathrm{A}^{-}+\mathrm{H}^{+} \\
& \mathrm{HOA} \stackrel{k_{7}}{\rightleftarrows} \mathrm{OA}^{-}+\mathrm{H}^{+} \\
& \mathrm{pyrOH} \stackrel{k_{8}}{\rightleftarrows} \mathrm{pyrO}^{-}+\mathrm{H}^{+}
\end{aligned}
$$


a) DIVISION

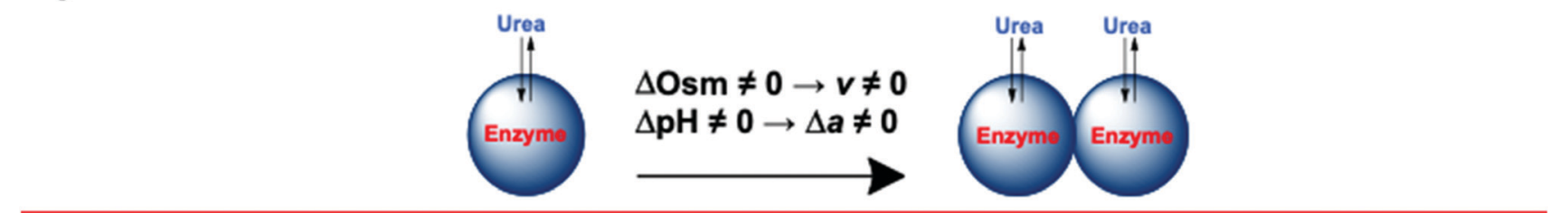

b)

$1 \mathrm{pH}$ buffer

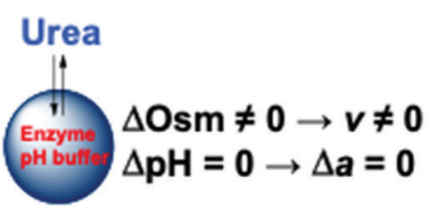

\section{Replacement of urea with neutral salts}

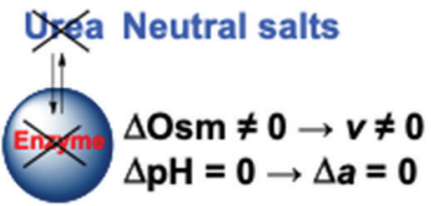

NO DIVISION

2 Absence of urea

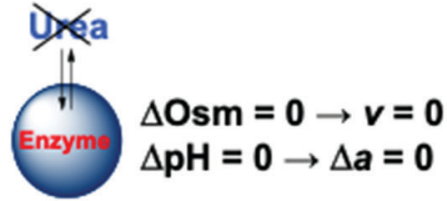

5 Elimination of osmotic gradient

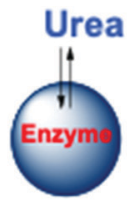

$\Delta$ Osm $=0 \rightarrow v=0$ $\Delta \mathrm{pH} \neq 0 \rightarrow \Delta a \neq 0$
3 Absence of enzyme Urea

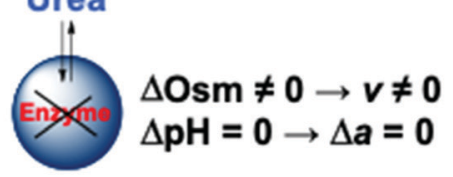

\section{$6 \mathrm{pH}$ change and osmolarity change are not synergic}

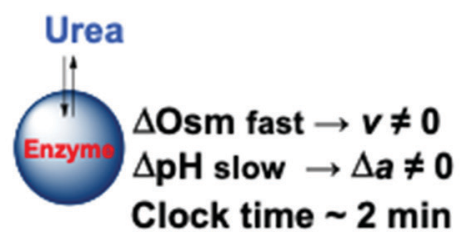

Fig. 2 Schematic representation of the control experiments with (a) and without (b) division.

The transfer of the chemical species across the membranes can be considered as a first order process described by the following reactions

$$
\begin{gathered}
\mathrm{S} \stackrel{k_{7}}{\rightleftharpoons} \mathrm{S}_{\text {out }} \\
\mathrm{NH}_{3} \stackrel{k_{\mathrm{N}}}{\rightleftharpoons} \mathrm{NH}_{3 \text { out }} \\
\mathrm{CO}_{2} \stackrel{k_{\mathrm{C}}}{\rightleftharpoons} \mathrm{CO}_{2 \text { out }} \\
\mathrm{HA} \stackrel{k_{\mathrm{HA}}}{\rightleftharpoons} \mathrm{HA}_{\text {out }}
\end{gathered}
$$

where $[\mathrm{X}]_{\text {out }}$ is the concentration of the chemical species $\mathrm{X}$ outside the vesicles in the water phase. The set of ordinary differential equations (ODEs) derived from the equilibria (6)(13) and (14)-(17) is reported in the ESI. $\dagger$

The ADE energy arises from the deviation in the total area difference $\Delta A$ from $\Delta A_{0}$. From a modelling point of view, it is clearer to report the shape transformation dynamics in terms of $\Delta A_{0}$ (or its reduced form $\Delta a_{0}$ ) which depends only on the number of amphiphilic molecules in the membrane and not on the vesicle shape. Therefore, the change of the number of molecules for oleic/oleate in the inner and outer leaflets (POPC molecules were constant) can be related to the $\mathrm{pH}$ dynamics described by equilibria (6)-(17).
In particular as $\mathrm{pH}$ increases, oleic acid molecules in the inner leaflet are deprotonated and can be solubilised as monomers or larger aggregates into the vesicle's lumen; the loss of oleate is, in turn, compensated by oleic acid molecules diffusing from the outer leaflet. The overall process is sketched in Fig. 3 and described by the following differential equations

$$
\begin{aligned}
& \frac{\mathrm{d} N_{\text {outer }}}{\mathrm{d} t}=\frac{\mathrm{d} N_{\text {outerPOPC }}}{\mathrm{d} t}+\frac{\mathrm{d} N_{\text {outerHOA }}}{\mathrm{d} t}+\frac{\mathrm{d} N_{\text {outerOA }^{-}}}{\mathrm{d} t} \\
& \frac{\mathrm{d} N_{\text {outer }}}{\mathrm{d} t}=-k_{\mathrm{f}}\left(N_{\text {outer }}-N_{\text {inner }} \frac{R_{\mathrm{s}}^{2}}{\left(R_{\mathrm{s}}-h\right)^{2}}\right) \\
& \frac{\mathrm{d} N_{\text {inner }}}{\mathrm{d} t}=\frac{\mathrm{d} N_{\text {innerPOPC }}}{\mathrm{d} t}+\frac{\mathrm{d} N_{\text {innerHOA }}}{\mathrm{d} t}+\frac{\mathrm{d}_{\text {innerOA }^{-}}}{\mathrm{d} t} \\
& \frac{\mathrm{d} N_{\text {inner }}}{\mathrm{d} t}=+k_{\mathrm{f}}\left(N_{\text {outer }}-N_{\text {inner }} \frac{R_{\mathrm{s}}^{2}}{\left(R_{\mathrm{s}}-h\right)^{2}}\right) \\
& -N_{\text {innerOA }}-\frac{k_{\text {off }}}{1+\mathrm{e}^{\left(-k_{\mathrm{t}}\left(\mathrm{pH}^{-} \mathrm{pH}_{\text {thres }}\right)\right)}}
\end{aligned}
$$

where $N_{\text {innerHOA }}$ and $N_{\text {innerOA }^{-}}$indicate the number of oleic acid molecules (unionized and deionized form) present in the inner leaflet, $N_{\text {outerHOA }}$ and $N_{\text {outerOA }^{-}}$are the oleic acid molecules in the outer leaflet. $R_{\mathrm{s}}$ is the radius of the initial spherical vesicle, $h$ is the distance between the two neutral surfaces. The number of molecules of POPC is considered constant $\left(\mathrm{d} N_{\text {outerPOPC }} / \mathrm{d} t=\right.$ $\left.\mathrm{d} N_{\text {innerPopc }} / \mathrm{d} t=0\right)$. The complete set of ODEs for the molecules of oleic acid can be found in the ESI. $\dagger$ 


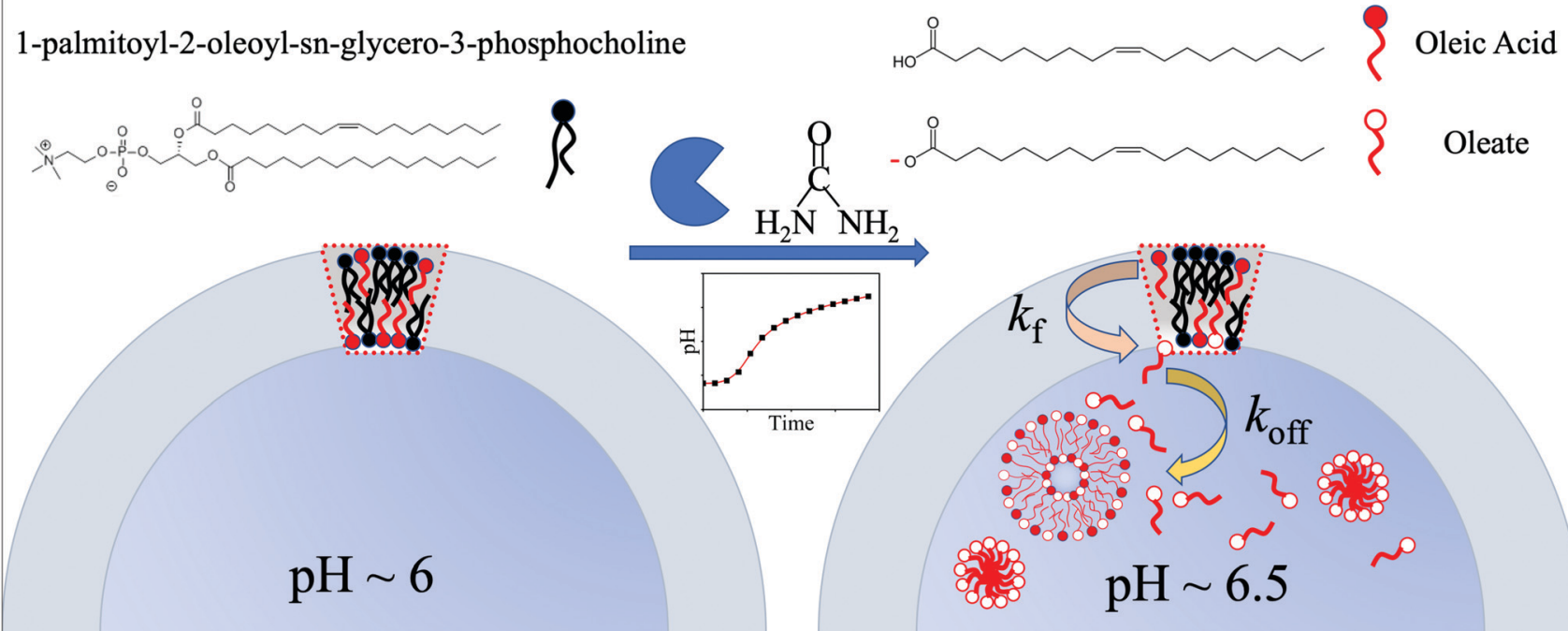

Fig. 3 Schematic representation of the mechanism of deformation. The enzymatic reaction leads to an increase of pH (from pH 6 to $\sim \mathrm{pH} 6.5$ ). Some $\mathrm{OA}^{-}$molecules of the inner leaflet are solubilized into the water pool (the solubility constant is $k_{\text {off }}$ ) and form vesicles, monomers and nonlamellar aggregates. HOA molecules diffuse from the outer to the inner leaflet with a kinetic constant $k_{\mathrm{f}}$.

As stated, the solubility of oleic acid in water solutions is $\mathrm{pH}-$ dependent, it increases with the $\mathrm{pH}$ undergoing abrupt transitions corresponding to different aggregation states (insoluble $\rightarrow$ oleate monomers $\rightarrow$ vesicles $\rightarrow$ micelles), ESR (electron spin resonance) measurements indicated a change in the aggregation (and solubility) properties of oleic acid at $\mathrm{pH} 6.3\left(\mathrm{pH}_{\text {thres }}\right.$ in eqn (21)), ${ }^{57}$ which is in the range of our experimental conditions. To account for such a behaviour we implemented the pH-dependency through a logistic function in the last term of eqn (21).

In order to compensate the dissolved molecules, oleic acid molecules diffuse from the outer leaflet to the inner leaflet with a rate constant $k_{\mathrm{f}}$ (the diffusion of the unionized form is usually higher compared to the ionized form ref. 58 and 59). The transport rate of fatty acids in eqn (19) and (21) takes into account the geometry of the vesicle: in a spherical vesicle, the number of outer molecules $\left(N_{\text {outer }}=4 \pi R_{\mathrm{s}}{ }^{2} /\langle\tilde{a}\rangle\right)$ is always higher than the number of inner molecules $\left(N_{\text {inner }}=4 \pi\left(R_{\mathrm{S}}-h\right)^{2} /\langle\tilde{a}\rangle\right)$ and consequently the relationship $N_{\text {outer }}=N_{\text {inner }} 4 \pi R_{\mathrm{s}}{ }^{2} / 4 \pi\left(R_{\mathrm{s}}-\right.$ $h)^{2}$ is respected ( $\langle\tilde{a}\rangle$ is the average cross section of a molecule). The analytical description of the $N_{\text {outer }} / N_{\text {inner }}=A_{\text {outer }} / A_{\text {inner }}$ is valid only for a spherical vesicle. However, we calculated this ratio for the different vesicle shapes assumed during the budding process (sphere $\rightarrow$ prolate $\rightarrow$ pear $\rightarrow$ double spheres); we extracted surface values from experimental snapshots to find that the ratio $A_{\text {outer }} / A_{\text {inner }}$ slightly varies from 1.005 to 1.009, passing from a sphere to a pear shape. When the solubilization constant is zero $N_{\text {outer }}=N_{\text {inner }} 4 \pi R_{\mathrm{s}}{ }^{2} / 4 \pi\left(R_{\mathrm{s}}-h\right)^{2}$ and $\mathrm{d} N_{\text {outer }} / \mathrm{d} t=0$. In contrast, when $k_{\text {off }}$ is different from zero, $N_{\text {outer }} \neq N_{\text {inner }} 4 \pi R_{\mathrm{s}}{ }^{2} / 4 \pi\left(R_{\mathrm{s}}-h\right)^{2}$, the outer oleic acid molecules will diffuse to compensate the dissolved oleate without removing the natural difference of molecules between the outer and the inner leaflet.

The number of molecules is correlated to the reference area of the membrane $\left(A_{0}\right)$, the preferred area difference $\left(\Delta A_{0}\right)$ and the normalized preferred area difference $\left(\Delta a_{0}\right)$

$$
\begin{gathered}
A_{0}(t)=\frac{N_{\text {outer }}(t)\langle\tilde{a}\rangle+N_{\text {inner }}(t)\langle\tilde{a}\rangle}{2} \\
\Delta A_{0}(t)=N_{\text {outer }}(t)\langle\tilde{a}\rangle-N_{\text {inner }}(t)\langle\tilde{a}\rangle \\
\Delta a_{0}(t)=\frac{\Delta A_{0}(t)}{8 \pi R_{0}(t) h}
\end{gathered}
$$

where $\langle\tilde{a}\rangle$ is the average cross section of the amphiphiles, $h$ is the distance between the neutral surfaces $(h \sim 2 \mathrm{~nm})$ and $R_{0(t)}=\sqrt{A_{0(t)} /(4 \pi)}{ }^{21}$ The average cross section at the beginning of the experiment $(\mathrm{pH} \sim 6)$ was calculated taking into account the molar fraction composition of the membrane and the single cross sections of POPC $\left(\tilde{a}_{\mathrm{POPC}}=0.69 \mathrm{~nm}^{2}\right)^{60}$ and oleic acid $\left(\tilde{a}_{\text {oleic }}=0.34 \mathrm{~nm}^{2}\right.$, for simplicity oleic and oleate have the same cross sectional area) ${ }^{61}$

$$
\langle\tilde{a}\rangle=\Phi \tilde{a}_{\mathrm{POPC}}+(1-\Phi) \tilde{a}_{\mathrm{oleic}}=0.512 \mathrm{~nm}^{2}
$$

Due to electrostatic interactions, the formation of oleate molecules increases the average cross section of the amphiphiles. ${ }^{60}$ For small $\mathrm{pH}$ intervals the increment can be considered linear to the $\mathrm{pH},\langle\tilde{a}\rangle_{t}=q+m \mathrm{pH}$ with $q=0.11$ and $m=0.066$, which account for an increase of $7 \%$ from $\mathrm{pH}=6$ to $\mathrm{pH}=6.5$ as determined by the Wilhelmy film balance experiments. ${ }^{30}$ The average cross section at $\mathrm{pH} 6$ is comparable with the values of average area per head group simulated at $\mathrm{pH}$ $5.5\left(0.54 \pm 0.01 \mathrm{~nm}^{2}\right)$ and $\mathrm{pH} 7.5\left(0.66 \pm 0.01 \mathrm{~nm}^{2}\right)$ for mixed vesicles Lyso-PC: oleic acid $=50 \%: 50 \% .{ }^{60}$ The diffusion constant $k_{\mathrm{f}}$ and the solubilisation constant $k_{\text {off }}$ were chosen in line with previous works ${ }^{37}$ and slightly changed to match the timescale of our experiments, i.e. $k_{\mathrm{f}}=0.4 \mathrm{~s}^{-1}$ and $k_{\mathrm{off}}=0.008 \mathrm{~s}^{-1}$. For simplicity, the diffusion constant $k_{\mathrm{f}}$ does not consider the 

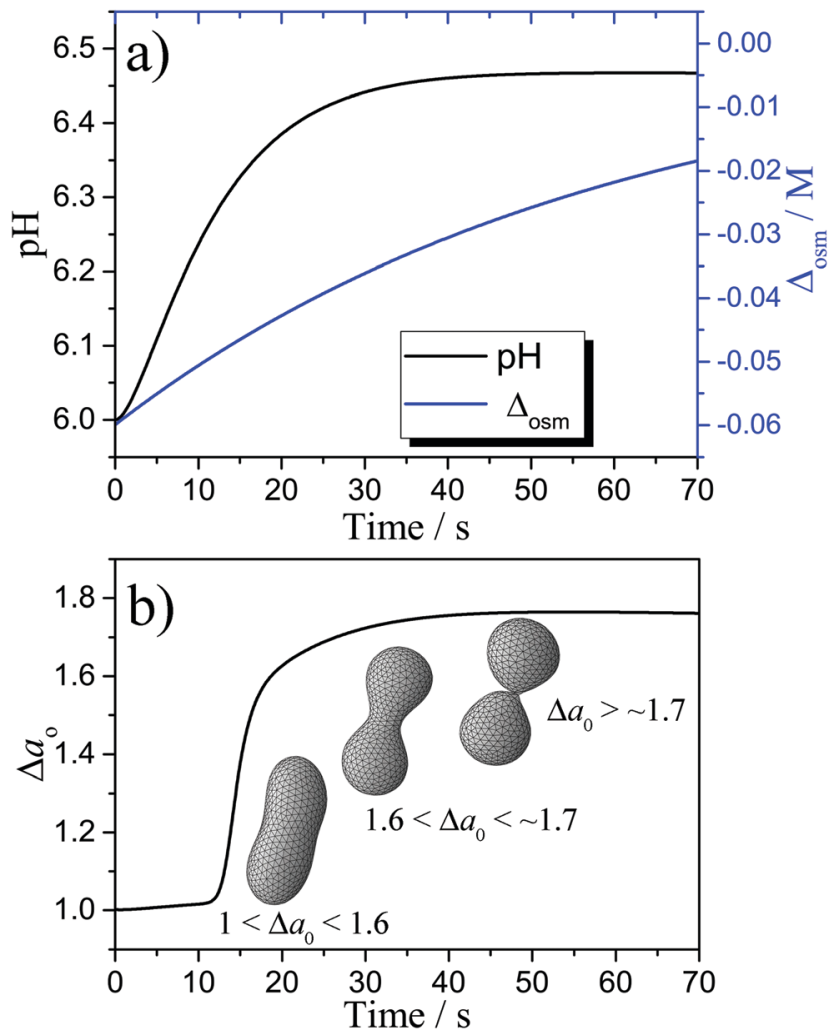

Fig. 4 (a) Simulated $\mathrm{pH}$ and $\Delta_{\text {osmi }}$ (b) simulated $\Delta a_{0}$ with the corresponding vesicle shape at $\nu=0.7$

dependence of the flip-flop rate from the vesicle shapes as predicted from molecular dynamics simulations. ${ }^{62}$ The values of the other relevant parameters (initial conditions, kinetic constants, etc.) are reported in the ESI. $\dagger$

Fig. 4(a) shows the simulated variation of the $\mathrm{pH}$ and the osmolarity $\left(\Delta_{\text {osm }}=\mathrm{osm}_{\text {in }}-\mathrm{osm}_{\text {out }}\right)$ inside a vesicle, osmolarity was calculated as the sum of the concentrations of the ionic species that cannot cross the membrane due to electroneutrality constrains, the concentrations of bulky molecules initially encapsulated into the vesicles (enzyme and pyranine) and the concentration of urea. The conversion of urea in $\mathrm{NH}_{3}$ and $\mathrm{CO}_{2}$ leads to a pH increase from $\sim 6$ to $\sim 6.4$ while the initial osmotic shock $\left(\Delta_{\text {osm }} \sim 60 \mathrm{mM}\right)$ is progressively smoothed out by the trans-membrane crossing of the urea from the outside aqueous medium to the lumen of the vesicle. The plots of Fig. 4 have been obtained with the kinetic model here proposed that combines the kinetics of the encapsulated reaction with the most important parameters of the ADE theory. Fig. 4(b) allows us to correlate the time scale of the $\mathrm{pH}$ variation with the time scale of the shape deformations. The variation of $\mathrm{pH}$ leads to the solubilisation of a certain amount of oleate that, when the buds are formed, can be estimated as the ratio between the dissolved oleic acid and the total molecules of oleic acid in the inner leaflet $(\sim 2 \%)$. Therefore, a small solubilization of oleate causes $\Delta a_{0}$ to reach the value of 1.8 in about $30 \mathrm{~s}$, corresponding to the limiting shape of two spherical vesicles connected by a narrow neck, ${ }^{63}$ as shown in Fig. 4(b).
For the sake of simplicity, the vesicle's shapes reported in Fig. 4(b) were calculated at the same reduced volume $\nu=0.7$, which was kept constant during the kinetic simulations.

We also performed simulations where we alternatively set $k_{\text {off }}$ and $k_{\mathrm{f}}$ equal to 0 . In the case of $k_{\text {off }}=0$ we could observe only a small growth of the inner leaflet surface, due to the electrostatic repulsion of the oleate groups. However, $\Delta a_{0}$ does not significantly diverge from 1 , i.e. the vesicle remains in a spherical form. When $k_{\mathrm{f}}=0$ none of the molecules from the outer leaflet replaces the solubilised oleate, therefore $\Delta a_{0}$ tends to reach unrealistic high values in the timeframe of the experiments. Both the diffusion of oleic acid between the leaflets and the solubilisation of the oleate in the lumen are important to drive the vesicles to a budded shape, but the latter is certainly the leading force of the mechanism. Diffusion of molecules from the outer to the inner leaflet is anyway important for preventing the vesicles to become too unstable.

\section{Conclusions}

The shape change leading to budding of a hybrid GUVs population was generated through a chemical stimulus inside the lumen (the $\mathrm{pH}$ change caused by the enzymatic reaction) and influenced by cross-membrane transport. The dynamics of changes driving the vesicles from a sphere to a budded limiting shape was explained in terms of an osmotic shock coupled to the $\mathrm{pH}$ change and rationalised with the support of the ADE theory, i.e. a variation of the number of amphiphilic molecules in the two leaflets of the membrane caused a deviation of $\Delta a_{0}$ from $\Delta a$. With the help of numerical simulations, the change of the number of amphiphiles has been explained by the dissolution of oleate into the vesicles' lumen and the concomitant diffusion of oleic acid molecules from the outer to the inner leaflet.

Both the $\mathrm{pH}$ and the osmotic pressure were previously exploited to control the shape transformation of GUVs. For example, a large variation of the external pH (5-6 units) was found to induce shape changes in pure fatty acid vesicles ${ }^{37}$ or changes of the preferred curvature of SOPC (1-stearoyl-2-oleoyl-phosphatidylcholine) membranes. ${ }^{64}$ In recent work, the proper control of the osmotic pressure induced the total division (two vesicles completely separated) in heterogeneous GUVs. ${ }^{65}$ In our system, the use of an internal chemical trigger and the presence of $\mathrm{pH}$-sensitive molecules in the membrane composition, allows us to attain membrane instabilities even for lower $\mathrm{pH}$ jumps ( 0.5 units), when this is coupled with an osmotic shock. The resemblance to living systems, in which the division is a process governed by the complex biochemical reaction network of the cell, often induced by environmental inputs sensed through crossmembrane transport, makes this system a plausible model for studying the self-division process of protocells under prebiotic conditions. ${ }^{66}$ Based on this knowledge, novel self-dividing systems can be designed having lifelike functions. 


\section{Conflicts of interest}

There are no conflicts of interest to declare.

\section{Acknowledgements}

This study was supported by the National Research, Development and Innovation Office of Hungary (NN125752 and K131425) and the NRDI Fund (TKP2020 IES, Grant No. BMEIE-NAT) based on the charter of bolster issued by the NRDI Office under the auspices of the Ministry for Innovation and Technology. Prof. Imre Derényi (Eötvös University) and Prof. Pasquale Stano (University of Salento) are gratefully acknowledged for fruitful discussions.

\section{Notes and references}

1 V. Noireaux, Y. T. Maeda and A. Libchaber, Proc. Natl. Acad. Sci. U. S. A., 2011, 108, 3473-3480.

2 E. D’Aguanno, E. Altamura, F. Mavelli, A. Fahr, P. Stano and P. L. Luisi, Life, 2015, 5, 969-996.

3 C. Xu, S. Hu and X. Chen, Mater. Today, 2016, 19, 516-532. 4 B. C. Buddingh and J. C. M. van Hest, Acc. Chem. Res., 2017, 50, 769-777.

5 E. Altamura, R. Fiorentino, F. Milano, M. Trotta, G. Palazzo, P. Stano and F. Mavelli, Biophys. Chem., 2017, 229, 46-56.

6 P. Stano, G. Rampioni, F. DAngelo, E. Altamura, F. Mavelli, R. Marangoni, F. Rossi and L. Damiano, Advances in Bionanomaterials, Springer, Cham, 2018, pp. 141-154.

7 Y. Miele, Z. Medveczky, I. Lagzi, M. A. Budroni and F. Rossi, in Artificial Life and Evolutionary Computation, ed. S. Cagnoni, M. Mordonini, R. Pecori, A. Roli and M. Villani, Springer International Publishing, Cham, 2019, pp. 138-150.

8 Y. Lyu, R. Peng, H. Liu, H. Kuai, L. Mo, D. Han, J. Li and W. Tan, Chem. Sci., 2020, 11, 631-642.

9 M. A. Budroni, K. Torbensen, S. Ristori, A. Abou-Hassan and F. Rossi, J. Phys. Chem. Lett., 2020, 11, 2014-2020.

10 J. Szostak, D. Bartel and P. Luisi, Nature, 2001, 409, 387-390.

11 P. L. Luisi, The Emergence of Life: From Chemical Origins to Synthetic Biology, Cambridge University Press, Cambridge, UK, 2006.

12 K. Ruiz-Mirazo, C. Briones and A. de la Escosura, Chem. Rev., 2014, 114, 285-366.

13 F. Mavelli, E. Altamura, L. Cassidei and P. Stano, Entropy, 2014, 16, 2488-2511.

14 A. Lopez and M. Fiore, Life, 2019, 9, 49.

15 G. Murtas, Mol. BioSyst., 2013, 9, 195-204.

16 T. Baumgart, S. T. Hess and W. W. Webb, Nature, 2003, 425, 821-824.

17 T. F. Zhu and J. W. Szostak, J. Am. Chem. Soc., 2009, 131, 5705-5713.

18 P. Peterlin, V. Arrigler, K. Kogej, S. Svetina and P. Walde, Chem. Phys. Lipids, 2009, 159, 67-76.

19 Y. Sakuma and M. Imai, Phys. Rev. Lett., 2011, 107, 198101. 20 Y. Sakuma and M. Imai, Life, 2015, 5, 651-675.
21 T. Jimbo, Y. Sakuma, N. Urakami, P. Ziherl and M. Imai, Biophys. J., 2016, 110, 1551-1562.

22 J. Dervaux, V. Noireaux and A. J. Libchaber, Eur. Phys. J. Plus, 2017, 132, 284.

23 M. Kurisu, H. Aoki, T. Jimbo, Y. Sakuma, M. Imai, S. Serrano-Luginbühl and P. Walde, Commun. Chem., 2019, 2(117), 1-10.

24 B. Novák and J. J. Tyson, Nat. Rev. Mol. Cell Biol., 2008, 9, 981-991.

25 K. Takakura, T. Toyota and T. Sugawara, J. Am. Chem. Soc., 2003, 125, 8134-8140.

26 K. Kurihara, M. Tamura, K.-I. Shohda, T. Toyota, K. Suzuki and T. Sugawara, Nat. Chem., 2011, 3, 775-781.

27 T. Litschel, B. Ramm, R. Maas, M. Heymann and P. Schwille, Angew. Chem., Int. Ed., 2018, 57, 16286-16290.

28 Y. Li and P. R. ten Wolde, Phys. Rev. Lett., 2019, 123, 148003.

29 H. R. Vutukuri, M. Hoore, C. Abaurrea-Velasco, L. van Buren, A. Dutto, T. Auth, D. A. Fedosov, G. Gompper and J. Vermant, Nature, 2020, 586, 52-56.

30 Y. Miele, Z. Medveczky, G. Hollo, B. Tegze, I. Derenyi, Z. Horvolgyi, E. Altamura, I. Lagzi and F. Rossi, Chem. Sci., 2020, 11, 3228-3235.

31 G. Hu, J. A. Pojman, S. K. Scott, M. M. Wrobel and A. F. Taylor, J. Phys. Chem. B, 2010, 114, 14059-14063.

32 F. Muzika, T. Bansagi, I. Schreiber, L. Schreiberová and A. F. Taylor, Chem. Commun., 2014, 50, 11107-11109.

33 B. Krajewska, J. Mol. Catal. B: Enzym., 2016, 124, 70-76.

34 Y. Miele, T. Bánsági, A. Taylor, P. Stano and F. Rossi, in Advances in Artificial Life, Evolutionary Computation and Systems Chemistry, ed. F. Rossi, F. Mavelli, P. Stano and D. Caivano, Springer International Publishing, 2016, pp. 197-208.

35 Y. Miele, T. Bánsági, A. Taylor and F. Rossi, in Advances in Bionanomaterials I, ed. S. Piotto, F. Rossi, S. Concilio, E. Reverchon and G. Cattaneo, Springer International Publishing, Cham, 2018, pp. 63-74.

36 V. Heinrich, S. Svetina and B. Žekš, Phys. Rev. E: Stat. Phys., Plasmas, Fluids, Relat. Interdiscip. Top., 1993, 48, 3112.

37 K. Ikari, Y. Sakuma, T. Jimbo, A. Kodama, M. Imai, P.-A. Monnard and S. Rasmussen, Soft Matter, 2015, 11, 6327-6334.

38 S. Pautot, B. J. Frisken and D. A. Weitz, Langmuir, 2003, 19, 2870-2879.

39 P. Carrara, P. Stano and P. L. Luisi, ChemBioChem, 2012, 13, 1497-1502.

40 P. Stano, F. Wodlei, P. Carrara, S. Ristori, N. Marchettini and F. Rossi, in Advances in Artificial Life and Evolutionary Computation, ed. C. Pizzuti and G. Spezzano, Springer International Publishing, 2014, pp. 58-74.

41 C. A. Schneider, W. S. Rasband and K. W. Eliceiri, Nat. Methods, 2012, 9, 671-675.

42 K. A. Brakke, Exper. Math., 1992, 1, 141-165.

43 A. Sakashita, N. Urakami, P. Ziherl and M. Imai, Soft Matter, 2012, 8, 8569-8581.

44 W. Helfrich, Phys. Lett. A, 1974, 50, 115-116.

45 S. Svetina and B. Žekš, The Anatomical Record, 2002, 268, 215-225. 
46 X. Bian, S. Litvinov and P. Koumoutsakos, Comput. Methods Appl. Mech. Eng., 2020, 359, 112758.

47 S. Svetina and B. Žekš, Eur. Biophys. J., 1989, 17, 101-111.

48 J. Käs and E. Sackmann, Biophys. J., 1991, 60, 825-844.

$49 \mathrm{~J} . \mathrm{N}$. Israelachvili, Intermolecular and surface forces, Academic press, 2011.

50 N. Bergstrand and K. Edwards, Langmuir, 2001, 17, 3245-3253.

51 L. Miao, U. Seifert, M. Wortis and H.-G. Döbereiner, Phys. Rev. E: Stat. Phys., Plasmas, Fluids, Relat. Interdiscip. Top., 1994, 49, 5389.

52 U. Seifert, Adv. Phys., 1997, 46, 13-137.

53 W. Wiese, W. Harbich and W. Helfrich, J. Phys.: Condens. Matter, 1992, 4, 1647.

54 P. Stano, D. T. P. Souza, P. Carrara, E. Altamura, E. DAguanno, M. Caputo, P. L. Luisi and F. Mavelli, Mech. Adv. Mater. Struct., 2015, 22, 748-759.

55 E. Altamura, P. Stano, P. Walde and F. Mavelli, Int. J. Unconvent. Comput., 2015, 11, 5-21.

56 C. François-Martin, J. E. Rothman and F. Pincet, Proc. Natl. Acad. Sci. U. S. A., 2017, 114, 1238-1241.

57 H. Fukuda, A. Goto, H. Yoshioka, R. Goto, K. Morigaki and P. Walde, Langmuir, 2001, 17, 4223-4231.
58 J. A. Hamilton, Prostaglandins, Leukotrienes Essent. Fatty Acids, 1999, 60, 291-297.

59 F. Kamp and J. A. Hamilton, Proc. Natl. Acad. Sci. U. S. A., 1992, 89, 11367-11370.

60 K. Lähdesmäki, O. S. Ollila, A. Koivuniemi, P. T. Kovanen and M. T. Hyvönen, Biochim. Biophys. Acta, Biomembr., 2010, 1798, 938-946.

61 P. Walde, T. Namani, K. Morigaki and H. Hauser, Liposome Technol., 2007, 1, 1-19.

62 H. Noguchi and M. Takasu, Phys. Rev. E: Stat., Nonlinear, Soft Matter Phys., 2001, 64, 041913.

63 N. Urakami, T. Jimbo, Y. Sakuma and M. Imai, Soft Matter, 2018, 14, 3018-3027.

64 H.-G. Döbereiner, G. Gompper, C. K. Haluska, D. M. Kroll, P. G. Petrov and K. A. Riske, Phys. Rev. Lett., 2003, 91, 048301.

65 Y. Dreher, K. Jahnke, E. Bobkova, J. P. Spatz and K. Göpfrich, Angew. Chem., Int. Ed., 2021, DOI: 10.1002/ anie.202014174.

66 H. W. H. v, B. J. H. M. Roekel, L. H. H. Rosier, P. A. J. Meijer, A. J. Hilbers and W. T. S. Markvoort, Huck and d. T. F. A. Greef, Chem. Soc. Rev., 2015, 44, 7465-7483. 